\title{
Reduction of radiation pneumonitis by V20-constraints in breast cancer
}

\author{
Ulla Blom Goldman ${ }^{1,5^{*}}$, Berit Wennberg ${ }^{2}$, Gunilla Svane ${ }^{3}$, Håkan Bylund ${ }^{4}$, Pehr Lind ${ }^{5}$
}

\begin{abstract}
Introduction: Adjuvant local-regional radiotherapy (LRRT) is routinely recommended for breast cancer patients. It is well known being related to pulmonary side-effects. We studied post-RT radiological changes on X-ray and $C T$, and correlated the findings with Quality of Life (QoL), common dosimetric factors and co-variates. The results were compared with a previously reported cohort of 137 irradiated women.

Methods: 88 women underwent chest X-ray and CT pre-and 4-5 months after 3-D planned LRRT, minimizing the dose to the ipsilateral lung to $V_{20}<30 \%$. The lung field was divided into 3 regions and the development of postRT density changes were graded (0-3). Patients with radiological changes were compared with non-responders. Clinical symptoms were registered and data on patient and treatment related co-variates were gathered prospectively. The ipsilateral lung dosimetric factors $V_{13}, V_{20}, V_{30}$ and mean dose were calculated and QoL was assessed before and 4 months after RT.
\end{abstract}

Results: The use of dose-volume constraints significally reduced moderate-severe radiological changes on chest X-ray compared with our earlier study (Chi square trend test: $p<0.001$ ). Symptomatic pneumonitis was also rare in the present study. No agreement was found between $C T$ and chest $X$-ray as diagnostic tools for post-RT pneumonitis. $V_{13}$ correlated independently with radiological changes on $C T$ (logistic regression: $p=0.04 ; R O C$ area: 0.7 ). The Co-variates smoking habits, age, chemotherapy, endocrine or trastuzumab therapy did not influence the outcome on multivariate analysis. QoL changes in physical function, i.e. fatigue, dyspnoea were not detected but there was a trend for a worse recovery after chemotherapy in patients with high $V_{13}$ (Spearman Rank Correlation: $p<0.05$ ).

Conclusions: The use of dose-volume constraints significantly reduced post-RT radiological changes on chest $X$-ray in LRRT for BC. The lung changes on CT were also generally limited when we used this strategy and was not always picked up on chest $X$-ray. Variation in $V_{13}$ alone was correlated with occurrence of lung changes on $C T$.

\section{Introduction}

Postoperative radiotherapy (RT) for breast cancer (BC) plays an important role for reducing the rates of local recurrence and death [1-3]. The treatment, however, delivers some unwanted irradiation to the lung and heart. Side-effects to the lungs are in the form of acute pneumonitis and sub acute/late lung fibrosis. The risk for acute and chronic RT-induced lung morbidity is influenced by total dose, dose per fraction and irradiated lung volume. When a 3-D RT-planning technique is used, it is possible to quantify and limit the amount of individually irradiated lung volume. Clinical data suggest that a total lung dose of more than 20 Gy given with conventional fractionation

\footnotetext{
* Correspondence: ulla.blom-goldman@karolinska.se

'Department of Oncology, Karolinska University Hospital, Stockholm, Sweden Full list of author information is available at the end of the article
}

should be avoided if the unirradiated lung volume is not sufficient to guarantee essential breathing function [4]. In our previous work, we found no case of moderate symptomatic radiation pneumonitis (RP) in patients who received doses $\geq 20$ Gy $\left(V_{20}\right)$ to less than $30 \%$ of the ipsilateral lung volume [5]. We therefore used this cut-off level in the present trial. Other groups have found relations between chemotherapy [6,7] and tamoxifen intake [8] and RT-induced lung toxicity. In previous studies we have also found an association with age $[5,9]$. Individual sensitivity to irradiation is also known but a rare genetic condition in the population[10]. However it is shown that possessions of specific genes variants is predictive for the development of adverse effects after radiotherapy [11-13]. In contrast smoking has been reported to reduce the risk of RTinduced pneumonitis [14]. Side-effects to the normal lung 
tissue can occur as early as 6 weeks from the start of RT with symptoms of fever, dyspnoea and cough [15]. Signs of interstitial pulmonary inflammation can be detected on chest radiography (X-ray) in the irradiated lung. A later phase with fibrosis can be detected from 20 weeks and after about 36 weeks stationary fibrosis is obtained [16,17].

This study was performed to evaluate radiological pneumonitis (RP) on X-ray and CT in irradiated breast cancer women when the lung dose-volume constraints of $\mathrm{V}_{20}<30 \%$ was used and to correlate the findings with common dosimetric factors (ipsilateral $\mathrm{V}_{13}, \mathrm{~V}_{20}$, $\mathrm{V}_{30}$, MLD), Quality of life (QoL)-effects, symptoms and co-variates and compare the outcome to a previously reported study of 137 irradiated women [9].

\section{Methods}

This study was approved by the local ethics committee. Participating women gave informed consent before study enrolment.

\section{Study population}

All women who were referred to the Radiotherapy Department at Stockholm Söder Hospital during 20032005 for adjuvant LRRT after surgery for early breast cancer were asked to participate in this trial. Ninety-five patients were included, but seven patients withdrew their consent due to early relapse and were not evaluable. Eighty-eight patients were thus followed for seven months after RT for symptoms of acute/subacute radiation induced pulmonary complication. Mastectomy was done in 69 patients, while 19 patients were operated with conservative breast surgery. Seventy-two patients were irradiated with LRRT to the chest wall or breast, axilla and supraclavicular region and in these patients the internal mammary lymph nodes (IMN) were included. A total of 16 patients received RT excluding the IMN, i.e. 9 patients were given irradiation to the breast, axilla and supraclavicular region and 7 patients were referred for RT to the axilla and supraclavicular fossa only.

The mean age of the patients was 56 years (range 32-81). Data on potential confounding factors were collected prospectively, i.e. history of cardio vascular or pulmonary co-morbidity, smoking habits, functional level (i.e. not being able to climb three flights of stairs without a rest due to shortness of breath) and adjuvant hormonal-, trastuzumab- and chemotherapy treatment.

The chemotherapy was concluded 3-4 weeks prior to RT. Concurrent chemotherapy was never given. The typically regime consisted of doxorubicin, cyclophosphamide and 5-fluorouracil, but in 28 patients the therapy included docetaxel. Five patients received trastuzumab during RT. Intake of tamoxifen and anastrozol during RT was evenly split among the women.
To asses and evaluate Quality of life (QoL) before and after RT we used the EORTC QLQ-C30 version 3.0 and the EORTC QLQ-BR23 questionnaires.

\section{Radiotherapy treatment techniques}

The used RT treatment techniques are described in detail in an earlier publication [18]. LRRT after mastectomy was delivered with an anterior electron beam covering the chest wall and the IMN (range 6-12 MeV) and with a $6 \mathrm{MV}$ photon beam covering the supraclavicular region. LRRT after partial mastectomy consisted of two tangential photon beams of 4 or $6 \mathrm{MV}$ including the breast parenchyma (50 Gy) and the regional lymph nodes were treated in a similar way as described above (46 Gy). An additional oblique electron beam was added to include the IMN (46 Gy) in four cases. The prescribed dose was given in daily fractions of $2 \mathrm{~Gy}$, five days a week. In the present study all patients underwent $3-\mathrm{D}$ dose treatment planning (Pinnacle; version $6.2 \mathrm{~b}$ ) with avoidance of a dose exciding 20 Gy to more than $30 \%$ of the ipsilateral lung volume but with a good coverage of the clinical target volume (CTV). The cumulative dose-volume histograms were calculated and the ipsilateral lung volume receiving $\geq 13$ Gy $\left(\mathrm{V}_{13}\right),>20$ Gy $\left(\mathrm{V}_{20}\right),>30 \mathrm{~Gy}\left(\mathrm{~V}_{30}\right)$ and mean lung dose were defined.

\section{Monitoring for symptomatic pneumonitis and evaluation of radiological pneumonitis on X-ray and CT with the Arrigada's classification}

All patients were followed for respiratory symptoms, i.e. cough, dyspnoea with or without fever, 1, 4 and 7 months after the termination of RT. The patients were classified into three groups according to CTC-criteria (version 2.0) [19].

0 . No complications: no registered respiratory symptoms monitored by the clinician.

1. Mild reaction: cough and/or dyspnoea with our without fever judged to be radiation induced.

2. Moderate reaction: same as 1 but with impaired daily functions and treated with corticosteroids.

CT of the thorax was performed before and 4 months after RT and standardized chest X-ray was conducted after 5 months and evaluated by the same specialist in diagnostic radiology (HB) as in our previous trial [5]. The reproducibility of this scoring system was validated in our earlier publication [5].

On the frontal chest radiograph the lung was divided into the three regions suggested by Arrigada, i.e. the apicallateral (A-L), central-parahilar (C-P) and basal-lateral (B-L) regions [19]. The border between the A-L and B-L regions was set at the level of the pulmonary artery. The width of the C-P region was set to $5 \mathrm{~cm}$ and the upper and lower border were set two vertebrae above and below the level of pulmonary artery, respectively. Radiological pneumonitis 
(RP) was quantified according to Arrigada's classification. The highest-density grade in each region, i.e. $0=$ no evidence of fibrosis, 1 = linear streaks, 2 = moderate opacification, 3 = complete opacification were added together to form total scores ranging from 0 to 9 . Total scores of 1-3 were considered to represent slight radiological RP and score of 4-9 moderate to severe RP. This method has been described in detail in our earlier study [5].

\section{Evaluation of Quality of life}

We used the European Organisation for Research and Treatment of Cancer (EORTC) form QLQ-30 (version 3.0) [20] and the EORTC QLQ-BR-23 to asses QoL [21]. The forms were completed at baseline prior to and 4 months after RT. The QLQ-30 questionnaires consist of a total of 30 items. Five functional scales (physical, role, cognitive, emotional and social); nine symptom scales (fatigue, nausea/vomiting, pain, dyspnea, insomnia, appetite loss, constipation, diarrhea and financial difficulties) and one Global health status. QLQ-BR23 includes 23 items assessing disease symptoms, therapy side effects such as breast, arm symptom, hair loss, body image, sexual functioning, sexual enjoyment and future perspective.

Eighty-one patients completed both measurements. In seven cases the pts did not receive the $2^{\text {nd }}$ form. The form was double-sided and in some cases not completed on the back page. Scoring was performed in according with the EORTC scoring manual. Statistics and missing data were handled according to the manual. A fourpoint response scale was used to asses each item concerning functions or symptoms from 1 (not at all) to 4 (very much), and a seven-point scale was used for global health status/QoL from 1 (very poor) to 7 (excellent). The scale scores were linearly transformed into scores of 0-100 according to the EORTC manual. A high score on the global health status/functional scale represents a high/healthy level of functioning. In contrast a high score on the symptom scale represents a high level of symptomatology/problems. A study of the subjective significance of changes in QoL scores has suggested that a mean change of 5 to 10 on the multi-item scales is perceived as little change, 10 to 20 as moderate change and greater than 20 as very much change. Greater than ten points on the transformed questionnaire scale were considered clinically meaningful [22,23].

In the present paper, three functional scales (physical functioning, role functioning and social functioning) and four symptom scales (fatigue, pain, dyspnoea and insomnia) were included from QLQ C-30. In the EORTC QLQ-BR23 form we included functional scales (future perspective).

\section{Statistical methods}

The relation between symptomatic and radiological RP and the relation between radiological $\mathrm{RP}$ and the dosimetric factors and co-variates was analyzed with univariate and multivariate logistic regression (Wald-Enter method). Chi square trend test was used for test of correlation between radiological RP on X-ray in the present and earlier studies. To test agreement between CT-and X-ray for the diagnosis of radiological RP, Kappa-statistics was used.

Receiver operating characteristics curves (ROC) were used to predict radiological RP with $V_{13}$ [24]. Changes in QoL-scores in relation with $\mathrm{V}_{13}$ were evaluated with Spearman Rank Correlation. All reported results were based on two-sided tests and p-values $<0.05$ were considered statistically significant.

\section{Results}

\section{Radiological and symptomatic radiation pneumonitis}

Figure 1 shows an example of post-RT radiological RP of grade 3 in the apical-lateral region of the left lung on chest X-ray (= total score 3 ). Symptomatic pneumonitis was very rare in this study. Only one patient developed a moderate reaction and was treated with corticosteroids and antibiotics, mild reactions were detected in 6 patients. There was, furthermore, no relation between symptomatic RP and radiological RP on chest X-ray or CT. Minor changes are not seen on chest X-ray, in due to that $\mathrm{CT}$ is a more sensitive method than X-ray to detect small effected areas of pulmonary changes.

The use of dose-volume constraints significally reduced moderate-severe radiological RP on X-ray compared with the earlier treatment series for the technique with LRRT + IMN (Chi square trend test $\mathrm{p}<0.001$ ) (Table 1). There was, however, no difference when we compared the outcome for the technique LRRT-IMN in

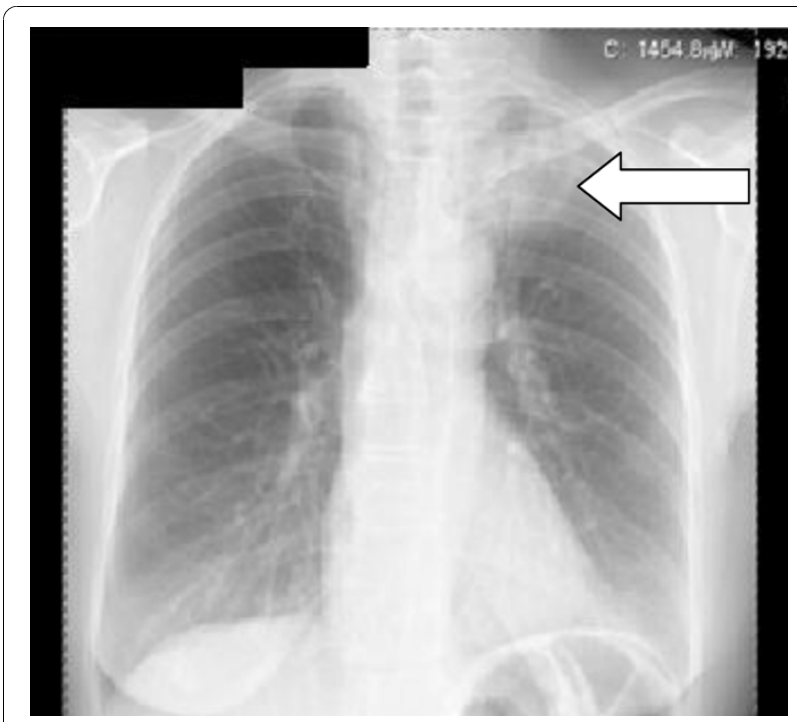

Figure 1 Example of grade $3 \mathrm{RP}$ in the apical-lateral region of the left lung on chest $\mathbf{x}$-ray. (= total score 3 ). 
Table 1 Relation between radiological changes and treatment techniques in the present and previous studies

\begin{tabular}{cccccccc}
\hline Technique & \multicolumn{3}{c}{ LRRT+ IMN; $\mathbf{n}$} & \multicolumn{3}{c}{ LRRT-IMN; $\mathbf{~}$} \\
\hline Arrigada's classification scores & 0 & $1-3$ & $4-9$ & 0 & $1-3$ & $4-9$ \\
\hline Present study & 60 & 11 & 1 & 5 & 4 & 0 \\
\hline Previous study & 58 & 38 & 20 & 12 & 9 & 0 \\
\hline & $\begin{array}{c}\text { Chi square trend } \\
\text { test } p<0.001\end{array}$ & $\begin{array}{c}\text { Chi square trend } \\
\text { test } p=0.9\end{array}$ \\
\hline
\end{tabular}

the present series with the previous trial (Table 1). The mean $\mathrm{V}_{20}$ for responders and non-responding patients are shown in Figure 2. The average $\mathrm{V}_{20}$ and MLD in our previous study was $35 \%$ and 16 Gy, respectively [25]. We found no correlation between any dosimetric factor or the studied co-variates and RP on chest X-ray (score 0 vs score 1-9) (logistic regression). In the preceding univariate analysis there was a borderline relation with radiological RP and anastrazol but this relation was thus not detected on the subsequent multivariate analysis which included the dosimetric factors and other co-variates.

There was no agreement between X-ray and CT as diagnostic tools for post-radiological RP, (Kappa test) (Table 2). $\mathrm{V}_{13}$ was most strongly and independently related with radiological changes on CT (score 0 vs 1-9) (logistic regression: $\mathrm{p}=0.04$; ROC-area: 0.7) [24]. No other factor was related to RP on CT. Table 3 shows the correlation between the dosimetric factors in this study. $V_{13}$ was stronger correlated to MLD than $V_{20}$.

\section{Quality of life}

Most of the side effects from RT appeared to have little effect on QoL in the present trial. Chemotherapy was concluded 3-4 weeks prior to RT and the patients started with a higher score on fatigue at baseline due to this.

The variables role functioning, social functioning and future perspective, were improved 4 months after RT compared to baseline (Table 4). Physical functioning,

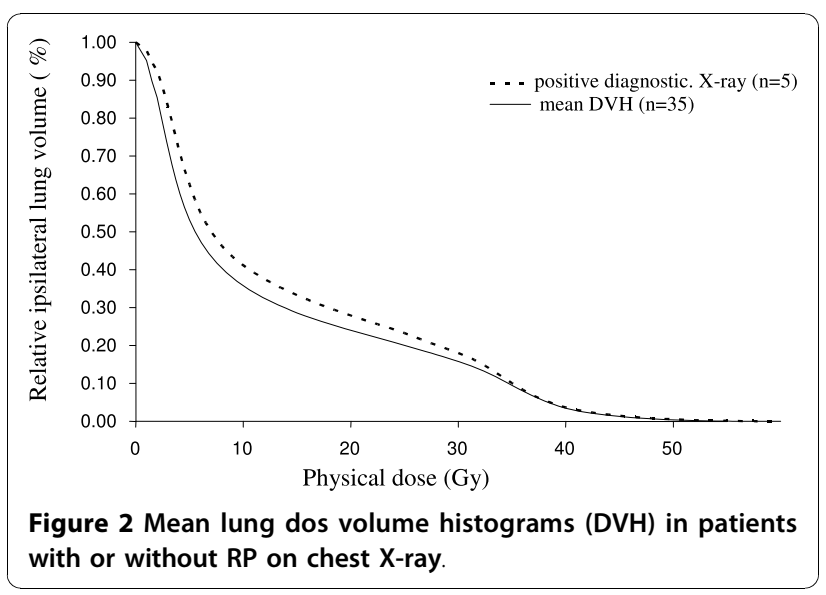

Table 2 Relation between radiological scores on X-ray and $C T$ in the present series

\begin{tabular}{ccccc}
\hline & \multicolumn{3}{c}{ CT score } & Total \\
\cline { 2 - 4 } X-ray score & $\mathbf{0}$ & $\mathbf{1 - 3}$ & $\mathbf{4 - 9}$ & 50 \\
\hline 0 & 13 & 25 & 12 & 11 \\
\hline $1-3$ & 1 & 7 & 3 & 1 \\
\hline $4-9$ & 0 & 1 & 0 & 62 \\
\hline Total & 14 & 33 & 15 & \\
\hline
\end{tabular}

Kappa statistics: $p=0.3$

appeared not to be affected by RT. There were no changes for pain and dyspnoea after RT in this series.

Patients with high $\mathrm{V}_{13}$ appeared however not recover equally well. However, insomnia showed a trend to increase after RT (Table 4). When changes in the individual QLQ-variates fatique and dyspnoea were related to $\mathrm{V}_{13}$ (Spearman correlation) there was thus a negative correlation. There was a significant correlation between high $\mathrm{V}_{13}$ and difficulties to take short walks, which could be of clinical significance, and the correlation was reported also when patients rated there overall total quality of life during the last week.

\section{Discussion}

Clinically significant radiological and symptomatic RP was rare in this study when 3-D treatment planning, aiming at minimizing $\mathrm{V}_{20}$ to the ipsilateral lung to $<30 \%$ was used for LRRT in early breast cancer. The result indicates that the used dose-volume constraints significantly reduced moderate-severe radiological RP on chest $\mathrm{X}$-ray, in the present series, compared to our previous study [25]. The lung changes could not always be detected on chest X-ray and were also infrequent and generally limited on CT when this strategy was used. Variation in dosimetry alone $\left(\mathrm{V}_{13}\right)$ was correlated with occurrence of radiological RP on CT. ROC analyses was performed, yet the area under the curve was only 0.7 which is not an ideal predictive value [24]. Co-variates

Table 3 Correlation between lung dosimetric factors in breast cancer irradiation

\begin{tabular}{llcccc}
\hline & & V13 & V20 & V30 & Mean \\
\hline \multirow{2}{*}{ V13 } & Pearsson Correlation & 1 & $.925^{* *}$ & $.619^{* *}$ & $.975^{* *}$ \\
& Sig. (2-tailed) & & .000 & .000 & .000 \\
\hline V20 & Pearsson Correlation & $.925^{* *}$ & 1 & $.820^{* *}$ & $.926^{* *}$ \\
& Sig. (2-tailed) & .000 & & .000 & .000 \\
\hline \multirow{2}{*}{ V30 } & Pearsson Correlation & $.619^{* *}$ & $.820^{* *}$ & 1 & $.687^{* *}$ \\
& Sig. (2-tailed) & .000 & .000 & & .000 \\
\hline Mean & Pearsson Correlation & $.975^{* *}$ & $.926^{* *}$ & $.687^{* *}$ & 1 \\
& Sig. (2-tailed) & .000 & .000 & .000 & \\
\hline
\end{tabular}

** Correlation is significant at the 0.01 level (2-tailed) 
Table 4 Pre and post RT Quality of Life EORTC scores in breast cancer irradiation

\begin{tabular}{|c|c|c|c|c|c|c|}
\hline & \multicolumn{6}{|c|}{ Mean QLQ-30 scale values before and after RT and for paired difference } \\
\hline & n & Before RT & After RT & Difference & $95 \% \mathrm{Cl}$ & P-value \\
\hline \multicolumn{7}{|l|}{ Functional scales } \\
\hline Physical functioning & 81 & 78.5 & 80.6 & 2.1 & $-1.2-5.4$ & 0.20 \\
\hline Role functioning & 80 & 62.3 & 70.6 & 8.3 & $1.3-15.4$ & 0.021 \\
\hline Social functioning & 73 & 70.6 & 76.3 & 5.7 & $0.7-10.7$ & 0.026 \\
\hline \multicolumn{7}{|l|}{ Symptom scales } \\
\hline Fatique & 73 & 37.1 & 31.7 & -5.5 & $-10.0--1.0$ & 0.018 \\
\hline Pain & 81 & 24.7 & 24.3 & -0.4 & $-5.3-4.4$ & 0.87 \\
\hline Dyspnoea & 79 & 29.1 & 28.3 & -0.8 & $-6.9-5.3$ & 0.78 \\
\hline \multirow[t]{3}{*}{ Insomnia } & 81 & 36.6 & 41.6 & 4.9 & $-1.5-11.3$ & 0.13 \\
\hline & \multicolumn{6}{|c|}{ Mean QLQ-BR23 scale values before and after RT and for paired difference } \\
\hline & $\mathbf{n}$ & Before RT & After RT & Difference & $95 \% \mathrm{Cl}$ & P-value \\
\hline \multicolumn{7}{|l|}{ Functional scales } \\
\hline Future perspective & 77 & 44.2 & 53.7 & 9.5 & 3.4-15.6 & 0.003 \\
\hline
\end{tabular}

as smoking habits, age, exposure to chemotherapy, endocrine- or trastuzumab therapy did not influence the outcome, but the few events may have hampered the possibility to evaluate this. In the present study, some women received radiation to the internal mammary nodes (IMN). Whether the IMN need to be included in the CTV is not fully known. In the last years, many RT centers have excluded RT to the lower IMN, in order to avoid cardiac and lung toxicity. The meta-analysis in Lancet, 2005, however demonstrated a benefit for post mastectomy RT in women with positive $\mathrm{LN}$ and the majority of these women received RT to the lower IMN (21 of 23 studies) [1].

The LRRT-IMN group of our present series included only nine patients. We used the same RT-technique in both this and the previous study, and as could be expected, there was no difference in radiological RP (Table 1). CTV volumes minus IMN usually give lower doses to the lung. Limiting the IMN irradiation to the three upper intercostals spaces also lower the dose to the heart. It is probably of great importance to reduce radiation to organs at risk like heart and lung, when adjuvant treatment is given. The average patient has a long expected survival, but as there are many new systemic therapies which may interact with RT this can lead to additional side-effects. Aromatase Inhibitors (AI) have replaced tamoxifen in many postmenopausal patients.

The AI treatment in combination with RT is investigated in a randomised trial presented in Lancet Oncol. 2010. The results suggested that AI can be used early, but there still are doubts on potential long-term toxic effects, mainly cardiac in combination with RT $[26,27]$.

Genetic factors may also play a vitale role in treatment. By identifying genetic factors associated with radiosensitivity it will be easier to predict which patients are at increased risk for complications secondary to radiation treatment [11-13].

Even though symptomatic and radiological RP were rare in our trial, they still could be of importance if they prevail, as late changes could increase the risk of secondary lung cancer. This increased risk is seen in smokers five years after RT [28].

To improve radiotherapy techniques and continue to study pulmonary morbidity and QoL after RT, is of great importance as breast cancer is a common disease among women.

In conclusion, V20-constraints significantly reduced post-RT radiological changes on chest X-ray in LRRT for breast cancer. Symptomatic pneumonitis was, furthermore, rare in the present study when this strategy was used. There was no agreement between X-ray and $\mathrm{CT}$ as diagnostic tools for post-RT in this trial, as the lung changes typically were too limited for detection on $\mathrm{X}$-ray. $\mathrm{V}_{13}$ was most strongly related to radiological RP on CT. $V_{13}$ was stronger correlated to MLD than $V_{20}$, and may be an important metric in future trials on RTinduced lung toxicity.

\section{Acknowledgements}

Presented at ASTRO 2009

This work was supported by The Swedish Cancer Foundation (Cancerfonden). We are grateful for the work that the staff of the Radiotherapy Department and the Breast Cancer Outpatient Ward at Stockholm Söder Hospital has put into this study.

\section{Author details}

'Department of Oncology, Karolinska University Hospital, Stockholm, Sweden. ${ }^{2}$ Department of Hospital Physics, Karolinska University Hospital, Stockholm, Sweden. ${ }^{3}$ Department of Radiology, Karolinska University Hospital, Stockholm, Sweden. ${ }^{4}$ Department of Radiology, Ersta Hospital, Stockholm, Sweden. ${ }^{5}$ Karolinska Institutet Stockholm, Sweden.

Authors' contributions

UBG coordinated the study, collected the data and drafted the manuscript. 
UBG, BW, GS and PL were involved with the design of the study. $\mathrm{HB}$ and GS analysed X-ray and CT diagnostics. BW analysed RT-doses. PL supported with the statistics. All authors read and approved the final manuscript.

\section{Competing interests}

The authors declare that they have no competing interests.

Received: 2 June 2010 Accepted: 29 October 2010

Published: 29 October 2010

\section{References}

1. Clarke M, Collins R, Darby S, Davies C, Elphinstone P, Evans E, Godwin J, Gray R, Hicks C, James S, et al: Effects of radiotherapy and of differences in the extent of surgery for early breast cancer on local recurrence and 15-year survival: an overview of the randomised trials. Lancet 2005, 366:2087-2106.

2. Overgaard M, Hansen PS, Overgaard J, Rose C, Andersson M, Bach F, Kjaer M, Gadeberg CC, Mouridsen HT, Jensen MB, Zedeler K: Postoperative radiotherapy in high-risk premenopausal women with breast cancer who receive adjuvant chemotherapy. Danish Breast Cancer Cooperative Group 82b Trial. N Engl J Med 1997, 337:949-955.

3. Overgaard $M$, Jensen MB, Overgaard J, Hansen PS, Rose C, Andersson M, Kamby C, Kjaer M, Gadeberg CC, Rasmussen BB, et al: Postoperative radiotherapy in high-risk postmenopausal breast-cancer patients given adjuvant tamoxifen: Danish Breast Cancer Cooperative Group DBCG 82c randomised trial. Lancet 1999, 353:1641-1648.

4. Herrman T, Schorcht J, V M: Late sequelae in oncology. In Berlin Edited by: Dunst J, Saueer R 1995.

5. Lind PA, Svane G, Gagliardi G, Svensson C: Abnormalities by pulmonary regions studied with computer tomography following local or localregional radiotherapy for breast cancer. Int I Radiat Oncol Biol Phys 1999, 43:489-496.

6. Lind PA, Marks LB, Jamieson TA, Carter DL, Vredenburgh JJ, Folz RJ, Prosnitz LR: Predictors for pneumonitis during locoregional radiotherapy in high-risk patients with breast carcinoma treated with high-dose chemotherapy and stem-cell rescue. Cancer 2002, 94:2821-2829.

7. Taghian AG, Assaad SI, Niemierko A, Kuter I, Younger J, Schoenthaler R, Roche M, Powell SN: Risk of pneumonitis in breast cancer patients treated with radiation therapy and combination chemotherapy with paclitaxel. J Natl Cancer Inst 2001, 93:1806-1811.

8. Bentzen SM, Skoczylas JZ, Overgaard M, Overgaard J: Radiotherapy-related lung fibrosis enhanced by tamoxifen. J Natl Cancer Inst 1996, 88:918-922.

9. Lind PA, Bylund H, Wennberg B, Svensson C, Svane G: Abnormalities on chest radiographs following radiation therapy for breast cancer. Eur Radiol 2000, 10:484-489.

10. Baumann M: Impact of endogenous and exogenous factors on radiation sequelae Berlin: Springer-Verlag; 1995.

11. Ho AY, Atencio DP, Peters S, Stock RG, Formenti SC, Cesaretti JA, Green S, Haffty B, Drumea K, Leitzin L, et al: Genetic predictors of adverse radiotherapy effects: the Gene-PARE project. Int I Radiat Oncol Biol Phys 2006, 65:646-655.

12. Ozsahin M, Crompton NE, Gourgou S, Kramar A, Li L, Shi Y, Sozzi WJ, Zouhair A, Mirimanoff RO, Azria D: CD4 and CD8 T-lymphocyte apoptosis can predict radiation-induced late toxicity: a prospective study in 399 patients. Clin Cancer Res 2005, 11:7426-7433.

13. Azria D, Ozsahin M, Kramar A, Peters S, Atencio DP, Crompton NE, Mornex F, Pelegrin A, Dubois JB, Mirimanoff RO, Rosenstein BS: Single nucleotide polymorphisms, apoptosis, and the development of severe late adverse effects after radiotherapy. Clin Cancer Res 2008, 14:6284-6288.

14. Bjermer $L$, Franzen $L$, Littbrand B, Nilsson $K$, Angstrom T, Henriksson R: Effects of smoking and irradiated volume on inflammatory response in the lung of irradiated breast cancer patients evaluated with bronchoalveolar lavage. Cancer Res 1990, 50:2027-2030.

15. Davis SD, Yankelevitz DF, Henschke Cl: Radiation effects on the lung: clinical features, pathology, and imaging findings. AJR Am J Roentgenol 1992, 159:1157-1164

16. Slanina J, Sigmund G, Hinkelbein W, Wenz W, Wannenmacher M: [Pulmonary reaction to radiation following mantle-field irradiation. Comparison of follow-up by conventional x-ray and by computed tomography]. Radiologe 1988, 28:20-28.
17. Lind P: Clinical relevance of pulmonary toxicity in adjuvant breast cancer irradiation. Acta Oncol 2006, 45:13-15.

18. Lind PA, Rosfors S, Wennberg B, Glas U, Bevegard S, Fornander T: Pulmonary function following adjuvant chemotherapy and radiotherapy for breast cancer and the issue of three-dimensional treatment planning. Radiother Oncol 1998, 49:245-254.

19. Arriagada $R$, de Guevara JC, Mouriesse $H$, Hanzen C, Couanet D, Ruffie P, Baldeyrou P, Dewar J, Lusinchi A, Martin M, et al: Limited small cell lung cancer treated by combined radiotherapy and chemotherapy: evaluation of a grading system of lung fibrosis. Radiother Oncol 1989, 14:1-8.

20. Aaronson NK, Ahmedzai S, Bergman B, Bullinger M, Cull A, Duez NJ, Filiberti A, Flechtner H, Fleishman SB, de Haes JC, et al: The European Organization for Research and Treatment of Cancer QLQ-C30: a qualityof-life instrument for use in international clinical trials in oncology. J Natl Cancer Inst 1993, 85:365-376.

21. Sprangers MA, Groenvold M, Arraras JI, Franklin J, te Velde A, Muller M, Franzini L, Williams A, de Haes HC, Hopwood P, et al: The European Organization for Research and Treatment of Cancer breast cancerspecific quality-of-life questionnaire module: first results from a threecountry field study. J Clin Oncol 1996, 14:2756-2768.

22. Osoba D, Rodrigues G, Myles J, Zee B, Pater J: Interpreting the significance of changes in health-related quality-of-life scores. J Clin Oncol 1998, 16:139-144.

23. Velikova G, Stark D, Selby P: Quality of life instruments in oncology. Eur J Cancer 1999, 35:1571-1580.

24. Lind PA, Wennberg B, Gagliardi G, Rosfors S, Blom-Goldman U, Lidestahl A, Svane G: ROC curves and evaluation of radiation-induced pulmonary toxicity in breast cancer. Int J Radiat Oncol Biol Phys 2006, 64:765-770.

25. Lind PA, Wennberg B, Gagliardi G, Fornander T: Pulmonary complications following different radiotherapy techniques for breast cancer, and the association to irradiated lung volume and dose. Breast Cancer Res Treat 2001, 68:199-210.

26. Azria D, Belkacemi Y, Romieu G, Gourgou S, Gutowski M, Zaman K, Moscardo CL, Lemanski C, Coelho M, Rosenstein B, et al: Concurrent or sequential adjuvant letrozole and radiotherapy after conservative surgery for early-stage breast cancer (CO-HO-RT): a phase 2 randomised trial. Lancet Oncol 2010, 11:258-265.

27. Recht A: Radiotherapy, antihormonal therapy, and personalised medicine. Lancet Oncol 2010, 11:215-216.

28. Prochazka M, Hall P, Gagliardi G, Granath F, Nilsson BN, Shields PG, Tennis $M$, Czene K: lonizing radiation and tobacco use increases the risk of a subsequent lung carcinoma in women with breast cancer: case-only design. J Clin Oncol 2005, 23:7467-7474.

doi:10.1186/1748-717X-5-99

Cite this article as: Goldman et al:: Reduction of radiation pneumonitis by V20-constraints in breast cancer. Radiation Oncology 2010 5:99.

\section{Submit your next manuscript to BioMed Central and take full advantage of:}

- Convenient online submission

- Thorough peer review

- No space constraints or color figure charges

- Immediate publication on acceptance

- Inclusion in PubMed, CAS, Scopus and Google Scholar

- Research which is freely available for redistribution 